\title{
A decrease in blood pressure is associated with unfavorable outcome in patients undergoing thrombectomy under general anesthesia
}

\author{
Kilian M Treurniet, ${ }^{1}$ Olvert A Berkhemer, ${ }^{1,2,3}$ Rogier V Immink, ${ }^{4}$ Hester $\mathrm{F}$ Lingsma, ${ }^{5}$ \\ Vivian M C Ward-van der Stam, ${ }^{4}$ Markus W Hollmann, ${ }^{4}$ Jaap Vuyk, ${ }^{6}$ \\ Wim $\mathrm{H}$ van Zwam, $^{3}$ Aad van der Lugt, ${ }^{7}$ Robert J van Oostenbrugge, ${ }^{8}$ \\ Diederik W J Dippel, ${ }^{2}$ Jonathan M Coutinho, ${ }^{9}$ Yvo B W E M Roos, ${ }^{9}$ \\ Henk A Marquering, ${ }^{1,10}$ Charles B L M Majoie, ${ }^{1}$ for the MR CLEAN investigators
}

For numbered affiliations see end of article.

\section{Correspondence to} Professor Charles B L M Majoie, C1-426, Department of Radiology, Academic Medical Center, PO Box 22660, Amsterdam 1100DD, The Netherlands; c.b.majoie@amc.uva.nl

Received 13 January 2017 Revised 1 March 2017 Accepted 3 March 2017 Published Online First 12 April 2017

CrossMark

To cite: Treurniet $\mathrm{KM}$, Berkhemer OA, Immink RV et al. J Neurolntervent Surg 2018:10:107-111

\section{ABSTRACT}

Background Up to two-thirds of patients are either dependent or dead 3 months after thrombectomy for acute ischemic stroke (AIS). Loss of cerebral autoregulation may render patients with AIS vulnerable to decreases in mean arterial pressure (MAP).

Objective To determine whether a fall in MAP during intervention under general anesthesia (GA) affects functional outcome.

Methods This subgroup analysis included patients from the MR CLEAN trial treated with thrombectomy under $\mathrm{GA}$. The investigated variables were the difference between MAP at baseline and average MAP during GA $(\triangle \mathrm{MAP})$ as well as the difference between baseline MAP and the lowest MAP during GA ( $\triangle L M A P)$. Their association with a shift towards better outcome on the modified Rankin Scale (mRS) after 90 days was determined using ordinal logistic regression with adjustment for prognostic baseline variables.

Results Sixty of the 85 patients treated under GA in MR CLEAN had sufficient anesthetic information available for the analysis. A greater $\triangle \mathrm{MAP}$ was associated with worse outcome (adjusted common OR (acOR) 0.95 per point $\mathrm{mm} \mathrm{Hg}, 95 \% \mathrm{Cl} 0.92$ to 0.99 ). An average MAP during GA $10 \mathrm{~mm} \mathrm{Hg}$ lower than baseline MAP constituted a 1.67 times lower odds of a shift towards good outcome on the mRS. For $\triangle \mathrm{LMAP}$ this association was not significant (acOR 0.97 per $\mathrm{mm} \mathrm{Hg}, 95 \% \mathrm{Cl} 0.94$ to $1.00, \mathrm{p}=0.09$ ).

Conclusions A decrease in MAP during intervention under GA compared with baseline is associated with worse outcome.

Trial registration number NTR1804;

ISRCTN10888758; post-results.

\section{BACKGROUND}

Although the efficacy of mechanical thrombectomy in patients with acute ischemic stroke (AIS) has been proved in multiple studies, ${ }^{1-7}$ two out of three treated patients are functionally dependent or dead (score on the modified Rankin Scale (mRS) >2) at 90 days. $^{3}$ It is unclear whether the use of general anesthesia (GA) in thrombectomy for AIS interacts with treatment effect. A recent meta-analysis found less favorable outcome in patients treated under GA. ${ }^{8}$ In a post hoc analysis of the Multicenter Randomized Clinical Trial of Endovascular Treatment for AIS in the Netherlands (MR CLEAN) cohort the significant treatment effect present in the total study population was also found in patients treated under local anesthesia, but not in patients treated under GA. ${ }^{9}$

Blood pressure levels during intervention could influence neurologic outcome in patients treated under GA. The large vessel occlusion required for thrombectomy in AIS that causes an AIS might also cause a loss of cerebral autoregulation. ${ }^{10}$ As a result, decreases in mean arterial pressure (MAP) might reduce cerebral blood flow, with subsequent further neurological deterioration.

This study aims to assess the association of a drop in blood pressure during intervention under GA compared with that at baseline with functional outcome in the MR CLEAN study.

\section{METHODS}

\section{Patients and data}

We performed a post hoc analysis of the MR CLEAN trial. In MR CLEAN, patients were randomized between usual care and usual care with additional IA treatment in those with confirmed proximal anterior circulation AIS. The inclusion and exclusion criteria for the MR CLEAN trial have been reported earlier. ${ }^{11}$ The decision about the type of anesthetic management during intervention was left to the discretion of the MR CLEAN centers and treating physicians. However, the majority of centers had a fixed protocol during the trial. ${ }^{9}$ No specific recommendations were provided by the steering committee about blood pressure management. Only patients treated under GA had peri-interventional blood pressure measurements available. Patients treated with endovascular therapy under GA were included in this subgroup analysis if their data satisfied the following quality criteria: 1. baseline blood pressure was available; 2. blood pressure data could be read/extracted from the anesthesia report; 3 . the induction anesthetic was listed in the report, and; 4 . one blood pressure registration had been made every $<10$ min during anesthesia (one gap of $15 \mathrm{~min}$ was allowed). This last interval was chosen as the intervals between the reported blood pressure measurements varied 
greatly between centers: from continuous arterial blood pressure measurements to measurements every 3, 5, or even $8 \mathrm{~min}$.

Baseline data (eg, National Institute of Health Stroke Scale (NIHSS) at presentation, time from onset to randomization, and baseline Alberta Stroke Program Early CT Score (ASPECTS)) were extracted from the MR CLEAN database. The baseline blood pressure was a single brachial measurement at admission to the emergency department at the recruiting center. Specific data concerning the anesthetic management during the intervention were extracted from the available anesthesiology reports. All data from the anesthesiology reports were collected from the start of anesthetic induction until awakening. In cases of prolonged anesthesia after the intervention, data collection stopped at the end of the intervention. Extracted data included the type of induction and maintenance anesthetic, blood pressure levels during the intervention, and use of blood pressure elevating medication (eg, norepinephrine). When both invasive and noninvasive blood pressure measurement was made, invasive measurement was used for data collection as this continuous measure could be more readily extracted at the proposed intervals. When a single systolic or diastolic measurement was missing, the value directly following the missing value was imputed.

From the systolic and diastolic blood pressure measurements (SBP and DBP, respectively), the MAP was calculated using the formula $\mathrm{MAP}=\mathrm{DBP}+1 / 3(\mathrm{SBP}-\mathrm{DBP})$. As our main measure of blood pressure decrease, we used $\triangle \mathrm{MAP}$ : the difference between baseline MAP and the average of all MAP values collected during GA at intervals of approximately $10 \mathrm{~min}$. Also, to assess the significance of large, small drops in MAP, $\triangle$ LMAP was used: the difference between MAP at baseline and the single lowest MAP during GA (figure 1). To correct for the baseline blood pressure level, both variables were also analyzed as a percentage of MAP at baseline. For all patients included in this analysis, written informed consent from patients or their representatives was obtained before randomization in the MR CLEAN study.

\section{Statistical analyses}

Our primary outcome measure is the score on the mRS at 90 days. This scale ranges from 0 ('no symptoms') to 6 ('dead'). Ordinal logistic regression is used to assess the association of the decrease in blood pressure with a shift towards better outcome on the mRS. The mRS was inversely coded (ie, level 0

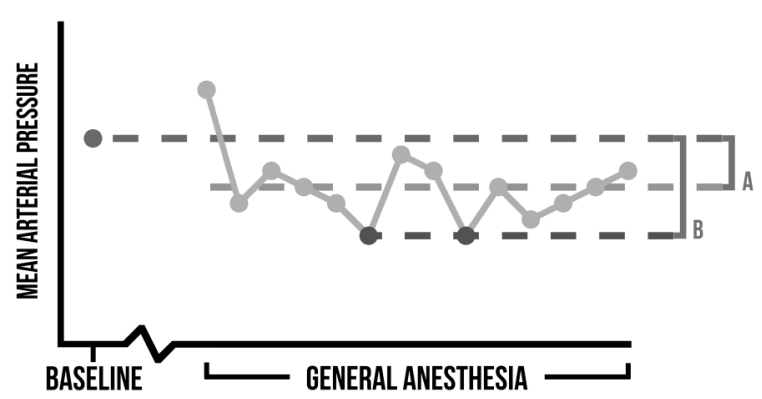

Figure 1 Schematic representation of the used blood pressure variables. The dot top left represents the baseline mean arterial pressure (MAP). Its level is represented by the upper dashed line. The line with dots represents the MAP values measured approximately every 10 min during general anesthesia (GA). Their average value is represented by the middle dashed line. The lowest dots and the lowest dashed line represent the lowest MAP value during GA. A represents the difference between baseline MAP and the average MAP during GA $(\triangle M A P)$. B represents the difference between baseline MAP and the lowest MAP during GA ( $\triangle \mathrm{LMAP})$. corresponds to mRS 6) so that a common OR (cOR) below 1 constitutes a drop in blood pressure that is associated with worse functional outcome. To adjust for known baseline prognostic variables as defined in the MR CLEAN protocol, ${ }^{11}$ the models include age, the baseline score on the NIHSS, history of atrial fibrillation, history of diabetes mellitus, previous stroke, presence of an internal carotid artery terminus occlusion, and time from onset to randomization.

Anesthetic agents are known to induce varying degrees of systemic hypotension.$^{12}$ Therefore, a univariable linear regression analysis was performed to see whether the type of anesthetic (propofol vs others) was associated with $\triangle \mathrm{MAP}$. As a longer procedure duration could yield more hypotensive events, its association with $\triangle$ MAP was determined in a similar manner. All statistics were performed with $\mathrm{R}$ ( $\mathrm{R}$ foundation for Statistical Computing, Vienna, Austria, http://www.r-project.org/; used packages: Ordinal v2015.6-28, http://CRAN.R-project.org/ package $=$ ordinal/; Foreign v0.8-63, http://CRAN.R-project.org/ package =foreign; Tableone v0.7.3, http://CRAN.R-project.org/ package=tableone; ggplot2 v2.2.0, http:/CRAN.R-project.org/ package $=$ ggplot2)

\section{RESULTS}

\section{Patients and characteristics}

Eighty-five patients underwent endovascular therapy under GA in the MR CLEAN trial. Six of those were converted from local anesthetic management, probably owing to increased movement. For this study, 15 patients were excluded because of partly or completely missing anesthesiology reports. Another 10 patients were excluded because the available data did not meet the predefined quality criteria. The remaining 60 patients were treated in nine different hospitals. Their characteristics are listed in table 1.

Table 1 Patient characteristics

\begin{tabular}{|c|c|}
\hline Characteristics & Value \\
\hline Number of patients & 60 \\
\hline Age, median (IQR) & $66(54-76)$ \\
\hline Time from onset to randomization (min), median (IQR) & $204(156-262)$ \\
\hline Sex, male, n (\%) & $35(58.3)$ \\
\hline \multicolumn{2}{|l|}{ Medical history, $\mathrm{n}(\%)^{*}$} \\
\hline Atrial fibrillation & $22(36.7)$ \\
\hline Diabetes mellitus & $7(11.7)$ \\
\hline Hypertension & $24(40.0)$ \\
\hline Ischemic stroke & $12(20.0)$ \\
\hline Myocardial infarction & $7(11.7)$ \\
\hline \multicolumn{2}{|l|}{ Occlusion location on admission CTA, $n(\%)$} \\
\hline$A 2$ & $1(1.7)$ \\
\hline ICA-T & $15(25.0)$ \\
\hline M1 & $39(65.0)$ \\
\hline $\mathrm{M} 2$ & $5(8.3)$ \\
\hline Admission NIHSS, median (IQR) & $18(14-20)$ \\
\hline Admission ASPECTS, median (IQR) $\dagger$ & $8.0(7.5-10)$ \\
\hline \multicolumn{2}{|l|}{ Admission blood pressure values (mm Hg), median (IQR) } \\
\hline SBP & $140(126-155)$ \\
\hline DBP & $80(70-90)$ \\
\hline MAP & $100(92-110)$ \\
\hline \multicolumn{2}{|c|}{$\begin{array}{l}\text { *Percentages may add up to more than } 100 \text { owing to comorbidity. } \\
\text { †As one patient had an A2 occlusion, ASPECTS is available for } 59 \text { patients. } \\
\text { ASPECTS, Alberta Stroke Program Early CT Score; CTA, CT angiography; DBP, diastolic } \\
\text { blood pressure; ICA-T, internal carotid artery terminus; MAP, mean arterial pressure; } \\
\text { NIHSS, National Institute of Health Stroke Scale; SBP, systolic blood pressure. }\end{array}$} \\
\hline
\end{tabular}


Data concerning anesthetic management are summarized in table 2.

Propofol was used most often to induce anesthesia $(n=45$, $75 \%)$ and sevoflurane was the compound most used to maintain anesthesia $(n=40,66.7 \%)$. In four cases midazolam was administered as premedication (dose $<5 \mathrm{mg}$ ) for induction with propofol $(n=3)$ or propofol and esketamine $(n=1)$, and in one case as inducing anesthetic (9 $\mathrm{mg}$ dose) combined with etomidate. Blood pressure elevating medication was administered in 54 of $60(90 \%)$ patients, but there was considerable variation in the type and combination. Thirty-six of the 54 (66.7\%) patients received norepinephrine. The median number of blood pressure measurements extracted from the anesthetic data was 11 (IQR 8-13) and the median procedure duration (groin puncture to end of procedure/sheath removal) was $69 \mathrm{~min}$ (IQR 47-96, data available for 59 of 60 patients). For four patients it was unclear

Table 2 Intraprocedural anesthetic data

\begin{tabular}{|c|c|}
\hline Anesthetic data & Value \\
\hline Number of patients & 60 \\
\hline \multicolumn{2}{|l|}{ Inducing Anesthesia, n (\%)* } \\
\hline Esketamine & $1(1.7)$ \\
\hline Etomidate & $8(13.3)$ \\
\hline Midazolam & $5(8.3)$ \\
\hline Propofol & $45(75.0)$ \\
\hline Thiopental & $7(11.7)$ \\
\hline \multicolumn{2}{|l|}{ Maintenance anesthetic, $\mathrm{n}(\%)^{*}$} \\
\hline None listed & $3(5.0)$ \\
\hline Isoflurane & $1(1.7)$ \\
\hline Propofol & $20(33.3)$ \\
\hline Sevoflurane & $40(66.7)$ \\
\hline \multicolumn{2}{|c|}{ Type of blood pressure elevating medication administered, $\mathrm{n}(\%)^{*}$} \\
\hline Ephedrine & $30(50.0)$ \\
\hline Norepinephrine & $36(60.0)$ \\
\hline Phenylephrine & $30(50.0)$ \\
\hline None & $6(10.0)$ \\
\hline \multicolumn{2}{|l|}{ Type of analgesic administered, $\mathrm{n}(\%)^{*}$} \\
\hline Acetominophen & $6(10.0)$ \\
\hline Alfentanil & $1(1.7)$ \\
\hline Fentanyl & $4(6.7)$ \\
\hline Lidocaine & $12(20.0)$ \\
\hline Metamizol & $1(1.7)$ \\
\hline Morphine & $1(1.7)$ \\
\hline Remifentanil & $11(18.3)$ \\
\hline Sufentanil & $42(70.0)$ \\
\hline None listed & $2(3.3)$ \\
\hline \multicolumn{2}{|c|}{ Blood pressure values during $\mathrm{GA}, \mathrm{mm} \mathrm{Hg}$, median (IQR) } \\
\hline SBP & $119(106-130)$ \\
\hline DBP & $64(59-71)$ \\
\hline MAP & $81(77-91)$ \\
\hline Lowest intraprocedural MAP & $60(55-69)$ \\
\hline \multicolumn{2}{|l|}{$\triangle \mathrm{MAP}$, median (IQR) } \\
\hline $\mathrm{mm} \mathrm{Hg}$ & $17(6.0-28)$ \\
\hline as $\%$ of admission blood pressure & $17(7.8-26)$ \\
\hline \multicolumn{2}{|l|}{$\triangle \mathrm{LMAP}$, median (IQR) } \\
\hline $\mathrm{mm} \mathrm{Hg}$ & $36(21-53)$ \\
\hline as $\%$ of admission blood pressure & $39(23-49)$ \\
\hline
\end{tabular}

whether the reported blood pressure was invasively or noninvasively measured, in 11 patients only invasive blood pressure measurements were available, and in 22 only non-invasive brachial measurements. For 23 patients, both non-invasive and invasive measurements were recorded during anesthesia, and measurements were extracted according to our prespecified methodology (ie, invasive when available). The median $\triangle \mathrm{MAP}$ was $17 \mathrm{~mm} \mathrm{Hg}$ (IQR 6-28) and $\triangle \mathrm{LMAP}$ $36 \mathrm{~mm} \mathrm{Hg}$ (IQR 21-53).

\section{Blood pressure decrease and outcome}

For all patients, their score on the mRS at 90 days and their respective $\triangle \mathrm{MAP}$ values are summarized in figure 2 . The results of the ordinal regression analyses are summarized in table 3 .

A greater $\triangle \mathrm{MAP}$ was associated with worse outcome (adjusted common OR (acOR) 0.95 per point $\mathrm{mm} \mathrm{Hg}$ decrease, 95\% CI 0.92 to 0.99 ).

This finding shows that for every $10 \mathrm{~mm} \mathrm{Hg}$ difference between baseline MAP and average MAP during GA the odds of a shift toward better outcome on the mRS are 1.67 times lower. For the $\triangle \mathrm{LMAP}$ no statistically significant association was found after the adjustment for baseline prognostic variables (acOR 0.97 per $\mathrm{mm} \mathrm{Hg}, 95 \%$ CI 0.94 to $1.00, \mathrm{p}=0.09$ ). For the adjusted analyses, findings were consistent when the variables were adjusted for the level of baseline MAP by expressing the size of the difference as the percentage of baseline MAP. No significant association was found between the use of propofol as an induction $(\beta=-8.49,95 \% \mathrm{CI}-18.00$ to 1.01$)$ or maintenance $(\beta=-4.98,95 \% \mathrm{CI}-13.85$ to 3.90$)$ anesthetic and $\triangle \mathrm{MAP}$. There was also no association between the procedure duration and $\triangle \mathrm{MAP}(\beta=0.02,95 \% \mathrm{CI}-0.09$ to 0.13$)$.

\section{DISCUSSION}

In this post hoc subgroup analysis in patients who received mechanical thrombectomy under GA during the MR CLEAN trial, we found that a decrease in peri-interventional MAP was associated with a worse functional outcome.

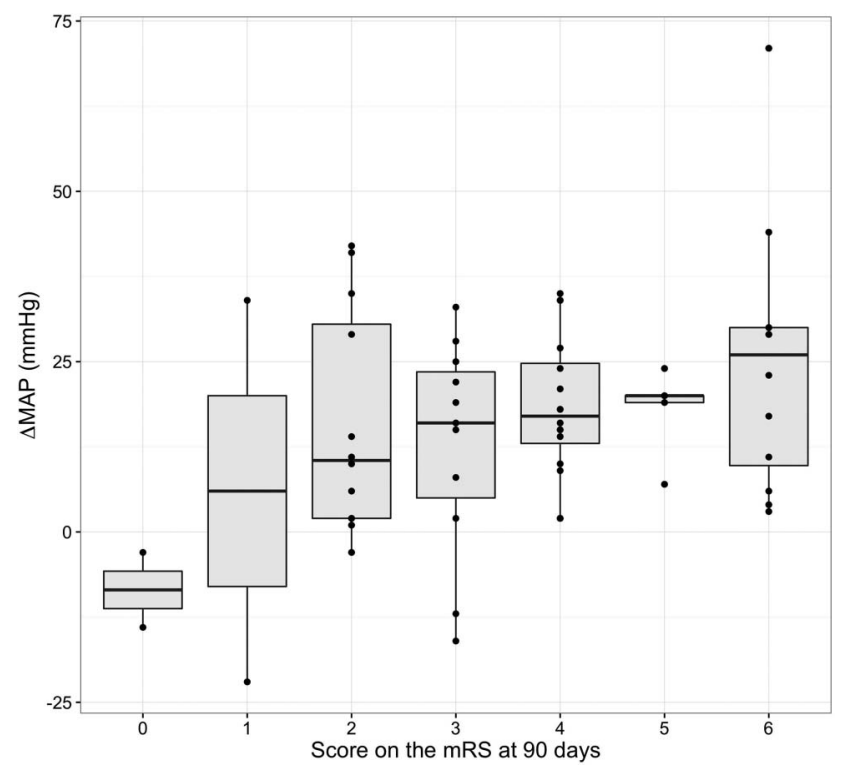

Figure 2 The association of the difference between baseline mean arterial pressure (MAP) and average MAP during GA ( $\triangle M A P)$ with the score on the modified Rankin Scale (mRS) at 90 days. The dots represent individual patients. The boxes depict the median (black bar) with the IQR. 
Table 3 Association of lower blood pressure during general anesthesia compared with baseline with a shift in the direction of better outcome on the modified Rankin Scale (mRS)

\begin{tabular}{|c|c|c|c|c|c|c|}
\hline \multirow[b]{2}{*}{ Variables } & \multicolumn{6}{|c|}{ Likelihood of a shift towards better outcome on $\mathrm{mRS}$} \\
\hline & COR & $95 \% \mathrm{Cl}$ & p Value & acOR & $95 \% \mathrm{Cl}$ & p Value \\
\hline$\triangle \mathrm{MAP}$, per unit $\mathrm{mm} \mathrm{Hg}$ & 0.96 & 0.93 to 0.99 & 0.01 & 0.95 & 0.92 to 0.99 & 0.01 \\
\hline As percentage of baseline MAP & 0.96 & 0.92 to 0.99 & 0.01 & 0.95 & 0.92 to 0.99 & 0.01 \\
\hline$\triangle \mathrm{LMAP}$, per unit $\mathrm{mm} \mathrm{Hg}$ & 0.97 & 0.95 to 1.00 & 0.03 & 0.97 & 0.94 to 1.00 & 0.09 \\
\hline As percentage of baseline MAP & 0.97 & 0.94 to 1.00 & 0.09 & 0.98 & 0.95 to 1.02 & 0.43 \\
\hline
\end{tabular}

While anesthetic management during IA therapy for AIS and the role of hemodynamic management therein has been discussed frequently, ${ }^{8}{ }^{12-16}$ few studies have specifically investigated the association of blood pressure with outcome in this setting. Our findings suggest that prolonged episodes of lower MAP compared with baseline during GA are potentially deleterious. Conversely, we found no significant association with outcome of the single largest MAP drop during GA after adjustment. Previous studies examined the occurrence of such single extreme blood pressure values. One did find significant associations with outcome, ${ }^{17}$ while others could not. ${ }^{18}{ }^{19}$ Another study investigating both patients under conscious sedation and GA found lower minimum diastolic blood pressures in patients with worse outcome. ${ }^{20}$ In a population consisting of both patients treated under GA and local anesthesia, a lowest systolic blood pressure of $<140 \mathrm{~mm} \mathrm{Hg}$ was found to be associated with poor outcome. ${ }^{21}$ The Society for Neuroscience in Anesthesiology and Critical Care recommends hemodynamic management with a systolic blood pressure $>140 \mathrm{~mm} \mathrm{Hg} .^{22}$ However, in patients with a subarachnoid hemorrhage, who have a similar loss of autoregulation in the brain, no significant association of hypotension with outcome was observed. ${ }^{23}$

As shown in our study, the use of a single extreme blood pressure variable (ie, the lowest MAP during GA) could not reproduce the same association as found when an averaged value of the intraprocedural MAP was used. Other studies also illustrate the dependency of results on the chosen variable. ${ }^{17} 20$ Therefore, the equivocal findings in the literature can partly be attributed to severe heterogeneity between studies in the investigated blood pressure variables.

Patients included in this study presented with a median admission systolic blood pressure of $140 \mathrm{~mm} \mathrm{Hg}$. Therefore, 50\% of included patients had admission blood pressure levels lower than the minimum level recommended by the Society for Neuroscience in Anesthesiology and Critical Care. ${ }^{22}$ Blood pressure levels were higher for patients not treated under GA in MR CLEAN (mean systolic blood pressure of $149 \mathrm{~mm} \mathrm{Hg}$ ), ${ }^{9}$ or patients in the intervention arm of other trials (median systolic blood pressures of $142-150 \mathrm{~mm} \mathrm{Hg}$ ). ${ }^{2}{ }^{4} 5$ However, these levels are still low in comparison with the finding that $76 \%$ of patients with AIS presented with a systolic blood pressure of $>140 \mathrm{~mm} \mathrm{Hg}^{24}$ We have no clear explanation for the low systolic blood pressure observed in this study. However, other baseline characteristics were very similarly distributed in comparison with the intervention arm of MR CLEAN. ${ }^{3}$ Also, the majority of MR CLEAN centers had a fixed protocol for the type of anesthetic management used. Blood pressure levels dropped even further during GA in our study. This can probably be attributed to a lack of awareness about the optimal anesthetic management for a treatment that was novel at the time inclusion in the MR CLEAN trial started.

This is the first study to assess the association of the difference between baseline MAP and average MAP during GA with continuous mRS. We specifically chose the MAP instead of the systolic or diastolic blood pressure as we feel it best approximates the perfusion pressure in the brain. As discussed above, the use of average intervention MAP is less prone to be influenced by measurement errors and short MAP fluctuations. Furthermore, the use of continuous mRS is likely to be more detailed than a dichotomized outcome variable as it takes the whole disability scale into account.

The study does have several limitations.

First, as this is a post hoc subgroup analysis, its results should be interpreted with caution, as the original study was not powered for these analyses.

Second, the multicenter design of MR CLEAN resulted in heterogeneous anesthesia data that had to be homogenized to make it suitable for analysis, as well as a large number of missing anesthesiology reports. This consequently led to a high exclusion rate (25 of 85 patients) to satisfy our predefined quality criteria.

Third, as reported earlier and seen in figure 2, only a few patients treated under GA in MR CLEAN were functionally independent after 3 months. ${ }^{9}$ Therefore at the lower end of the mRS spectrum only few blood pressure data are available. This also prevented us from determining a reliable estimate of a blood pressure threshold for poor outcome.

Fourth, the baseline blood pressure variable is based on a single measurement and is therefore more prone to measurement error.

Fifth, as blood pressure levels during GA for most patients were lower than the currently advised minimum of $140 \mathrm{~mm} \mathrm{Hg}$ systolic, it was not possible to examine whether adherence to this minimum blood pressure level was beneficial. ${ }^{22}$

Sixth, while the average MAP during intervention is more precise than a single extreme value, it does not take into account blood pressure variability.

Seventh, the comparison of baseline non-invasively measured blood pressure and intraprocedural invasive blood pressure measurements might have reduced the precision of the study. As shown in a large retrospective study, brachial measurements underestimate hypertensive blood pressure levels and produce higher values at the lower end of the spectrum. ${ }^{25}$ As hypertension is common in patients presenting with an AIS, ${ }^{24}$ this discrepancy might result in an underestimation of smaller drops in blood pressure in patients in whom intraprocedural invasive blood pressure measurements were extracted. Further, in the patients in whom both invasive and non-invasive measurements 
were collected this might have led to imprecise estimation of the true average MAP.

Eighth, patients in this study were not randomized to GA or alternative managements. Thus one cannot exclude the presence of any bias toward worse outcome.

Finally, the small sample size led to the inclusion of more variables in the regression models than the 10 events per variable that rule of thumb would allow. We deviated from this rule of thumb to allow for adequate correction for potential confounders, as was justified by Vittinghoff and McCulloch. ${ }^{26}$

The management of blood pressure is also of great importance for patients not treated under GA. Complex, u-curved relationships with outcome have been observed in patients with AIS, ${ }^{27} 28$ illustrating the delicate line between hyperperfusion predisposing to extensive cerebral edema, and hypoperfusion predisposing to infarct growth. Further, falls in blood pressure also seem to predispose to worse outcomes in patients undergoing intervention under conscious sedation. ${ }^{29}$ In this light, one might even postulate that the lack of difference in outcome between the GA and conscious sedation groups in the recently published Sedation versus Intubation for Endovascular Stroke TreAtment (SIESTA, NCT02126085) trial can partly be explained by the lack of difference in blood pressure levels between the trial arms. ${ }^{30}$

The SIESTA trial was the first completed trial investigating the place of GA in thrombectomy, and we await the results of at least two more: General Or Local Anesthesia in IA THerapy (GOLIATH, NCT02317237), ${ }^{31}$ and Sedation Versus GA for Endovascular Therapy in Acute Stroke-Impact on Neurological Outcome (ANSTROKE, NCT01872884). Post hoc analyses of their prospectively collected blood pressure data would yield more clarity about the association of blood pressure with outcome in and outside of a GA setting, and would provide some necessary guidance to improve outcome for patients with AIS even further.

\section{CONCLUSION}

Decreased MAP during intervention under GA compared with baseline is associated with worse outcome. However, prospective and systematically collected data are necessary to confirm this association.

\section{Author affiliations \\ 'Department of Radiology, Academic Medical Center, Amsterdam, The Netherlands ${ }^{2}$ Department of Neurology, Erasmus MC University Medical Center, Rotterdam, The Netherlands \\ ${ }^{3}$ Department of Radiology, Maastricht University Medical Center, Maastricht, The Netherlands \\ ${ }^{4}$ Department of Anesthesiology, Academic Medical Center, Amsterdam, The Netherlands \\ ${ }^{5}$ Department of Public Health, Erasmus MC University Medical Center, Rotterdam, The Netherlands \\ ${ }^{6}$ Department of Anesthesiology, Leiden University Medical Center, Leiden, The Netherlands \\ ${ }^{7}$ Department of Radiology, Erasmus MC University Medical Center, Rotterdam, The Netherlands \\ ${ }^{8}$ Department of Neurology, Maastricht University Medical Center, Maastricht, The Netherlands \\ ${ }^{9}$ Department of Neurology, Academic Medical Center, Amsterdam, The Netherlands ${ }^{10}$ Department of Biomedical Engineering \& Physics, Academic Medical Center, Amsterdam, The Netherlands}

Collaborators Executive committee: Diederik W J Dippel ${ }^{1}$; Aad van der Lugt ${ }^{2}$; Charles B L M Majoie; ; Yvo B W E M Roos ${ }^{4}$; Robert J van Oostenbrugge ${ }^{5}$; Wim H van Zwam ${ }^{6}$; Olvert A Berkhemer ${ }^{1,3}$; Puck S S Fransen ${ }^{1,2}$; Debbie Beumer ${ }^{1,5}$; Lucie A van den Berg ${ }^{4}$. Local principal investigators: Wouter J Schonewille; ; Jan Albert Vos ${ }^{8}$; Charles B L M Majoie; ${ }^{3}$ Y Yvo B W E M Roos ${ }^{4}$; Paul J Nederkoorn'; Marieke J H Wermer $^{9}$; Marianne A A van Walderveen ${ }^{10}$; Robert J van Oostenbrugge ${ }^{5}$; Wim $\mathrm{H}$ van
Zwam $^{6}$; Julie Staals ${ }^{5}$; Jeannette Hofmeijer ${ }^{11}$; Jacques A van Oostayen ${ }^{12}$; Geert J Lycklama à Nijeholt ${ }^{13}$; Jelis Boiten ${ }^{14}$; Diederik W J Dippel ${ }^{1}$; Patrick A Brouwer ${ }^{2}$; Bart J Emmer ${ }^{2}$; Sebastiaan F de Bruijn ${ }^{15}$; Lukas C van Dijk ${ }^{16}$; L Jaap Kappelle ${ }^{17}$; Rob H $\mathrm{Lo}^{18}$; Ewoud J van Dijk ${ }^{19}$; Joost de Vries ${ }^{20}$; Paul L M de Kort' ${ }^{21}$; Jan S P van den Berg $^{22}$; Willem Jan J van Rooij ${ }^{22}$; Boudewijn A A M van Hasselt ${ }^{23}$; Leo A M Aerden ${ }^{24}$; René J Dallinga ${ }^{25}$; Marieke C Visser ${ }^{26}$; Joseph C J Bot ${ }^{27}$; Patrick C Vroomen $^{28}$; Omid Eshghi ${ }^{29}$; Tobien H C M L Schreuder ${ }^{30}$; Roel J J Heijboer ${ }^{31}$; Koos Keizer $^{32}$; Alexander V Tielbeek ${ }^{33}$; Heleen M den Hertog ${ }^{34}$; Dick G Gerrits ${ }^{35}$; Renske $M$ van den Berg-Vos ${ }^{36} ;$ Giorgos B Karas ${ }^{37}$. Imaging assessment committee: Charles B L M Majoie ${ }^{3}$ (chair); Wim H van Zwam ${ }^{6}$; Aad van der Lugt'; Geert J Lycklama à Nijeholt ${ }^{13}$; Marianne A A van Walderveen ${ }^{10}$, Joseph C J Bot ${ }^{27}$; Henk A Marquering ${ }^{38}$; Ludo F Beenen ${ }^{3}$; Marieke E S Sprengers ${ }^{3}$; Sjoerd F M Jenniskens ${ }^{39}$, René van den Berg ${ }^{3}$; Olvert A Berkhemer ${ }^{1,3}$; Albert $\mathrm{J} \mathrm{YoO}^{40}$. Outcome assessment committee: Yvo B W E M Roos ${ }^{4}$ (chair); Peter J Koudstaal'; Jelis Boiten; Ewoud J. van Dijk ${ }^{19}$. Adverse event committee: Robert J van Oostenbrugge ${ }^{5}$ (chair); Marieke J H Wermer ${ }^{9}$; H Zwenneke Flach ${ }^{23}$. Trial statisticians: Ewout W Steyerberg ${ }^{41}$; Hester F Lingsma $^{41}$. List of affiliations: Department of Neurology ${ }^{1}$, Radiology ${ }^{2}$, Public Health ${ }^{41}$, Erasmus MC University Medical Center, Rotterdam; Department of Radiology ${ }^{3}$, Neurology ${ }^{4}$, Biomedical Engineering and Physics ${ }^{38}$, Academic Medical Center, Amsterdam; Department of Neurology ${ }^{5}$, Radiology ${ }^{6}$, Maastricht University Medical Center and Cardiovascular Research Institute Maastricht (CARIM); Department of Neurology ${ }^{7}$, Radiology ${ }^{8}$, Sint Antonius Hospital, Nieuwegein; Department of Neurology ${ }^{\prime}$, Radiology ${ }^{10}$, Medical Statistics and Bioinformatics ${ }^{44}$, Leiden University Medical Center; Department of Neurology ${ }^{11}$, Radiology ${ }^{12}$, Rijnstate Hospital, Arnhem; Department of Radiology ${ }^{13}$, Neurology ${ }^{14}$, MC Haaglanden, the Hague; Department of Neurology ${ }^{15}$, Radiology 16 , HAGA Hospital, the Hague; Department of Neurology ${ }^{17}$, Radiology ${ }^{18}$, University Medical Center Utrecht; Department of Neurology ${ }^{19}$, Neurosurgery ${ }^{20}$, Radiology ${ }^{39}$, Radboud University Medical Center, Nijmegen; Department of Neurology ${ }^{21}$, Sint Elisabeth Hospital, Tilburg; Department of Neurology 22 , Radiology ${ }^{23}$, Isala Klinieken, Zwolle; Department of Neurology ${ }^{24}$,

Radiology ${ }^{25}$, Reinier de Graaf Gasthuis, Delft; Department of Neurology ${ }^{26}$,

Radiology ${ }^{27}$, VU Medical Center, Amsterdam; Department of Neurology ${ }^{28}$

Radiology ${ }^{29}$, University Medical Center Groningen, the Netherlands; Department of Neurology ${ }^{30}$, Radiology ${ }^{31}$, Atrium Medical Center, Heerlen; Department of Neurology ${ }^{32}$, Radiology ${ }^{33}$, Catharina Hospital, Eindhoven; Department of Neurology ${ }^{34}$, Radiology35, Medical Spectrum Twente, Enschede; Department of Neurology $y^{36}$, Radiology 3 , Sint Lucas Andreas Hospital, Amsterdam; all in the Netherlands; Department of Radiology ${ }^{40}$, Texas Stroke Institute, Texas, USA.

Contributors KMT collected and cleaned data for this study, wrote the statistical analysis plan, analyzed the data, and drafted and revised the paper. He is guarantor. OAB implemented the MR CLEAN trial in the Netherlands, collected and cleaned the data for the trial and this study, and revised the draft paper. RVI, JV, and VMCW-vdS helped with the collection of data for this study and revised the draft paper. MWH and JMC revised the draft paper. HFL helped with the statistical analysis plan and revised the draft paper. RJvO, WHVZ, DWJD, AvdL and YBWEMR designed the MR CLEAN trial, implemented the trial in the Netherlands, oversaw data collection, and revised the draft paper of this study. HAM helped with the statistical analysis plan and drafted and revised the draft paper. CBLMM designed the MR CLEAN trial, implemented the trial in the Netherlands, oversaw data collection, planned this study, and drafted and revised the paper. The MR CLEAN investigators (listed in the appendix) collected the data for the MR CLEAN trial.

Funding The MR CLEAN trial was partly funded by the Dutch Heart Foundation grant number: 2008T30 and by unrestricted grants from AngioCare BV, Medtronic/ Covidien/EV3, MEDAC Gmbh/LAMEPRO, Penumbra Inc, Stryker, and Top Medical/ Concentric. The sponsors of the MR CLEAN trial were not involved in the design of the study, writing of the protocol, study conduct, or preparation or review of the article. The members of the executive committee and local investigators of the participating centers alone performed these tasks.

Competing interests Erasmus MC received funds from Stryker and Bracco Imaging for consultations by DWJD and AvdL. AMC received funds from Stryker for consultations by CBLMM, YBWEMR, and OAB. MUMC received funds from Stryker and Codman for consultations by WHvZ.

HAM is co-founder and shareholder of Nico-Lab.

Provenance and peer review Not commissioned; externally peer reviewed.

\section{REFERENCES}

1 Campbell BC, Mitchell PJ, Kleinig TJ, et al. Endovascular therapy for ischemic stroke with perfusion-imaging selection. N Engl J Med 2015;372: 1009-18.

2 Goyal M, Demchuk A, Menon B, et al. Randomized assessment of rapid endovascular treatment of ischemic stroke. N Engl J Med 2015;372:1019-30.

3 Berkhemer OA, Fransen PSS, Beumer D, et al. A randomized trial of intraarterial treatment for acute ischemic stroke. N Engl J Med 2015;372:11-20.

4 Jovin TG, Chamorro A, Cobo E, et al. Thrombectomy within 8 hours after symptom onset in ischemic stroke. N Engl J Med 2015;372:2296-306. 
5 Saver J, Goyal M, Bonafe A, et al. Stent-retriever thrombectomy after intravenous t-PA vs. t-PA alone in stroke. N Engl J Med 2015;372:2285-95.

6 Bracard S, Ducrocq X, Mas JL, et al. Mechanical thrombectomy after intravenous alteplase versus alteplase alone after stroke (THRACE): a randomised controlled trial. Lancet Neurol 2016;15:1138-47.

7 Goyal M, Menon BK, van Zwam WH, et al. Endovascular thrombectomy after large-vessel ischaemic stroke: a meta-analysis of individual patient data from five randomised trials. Lancet 2016:387:1723-31.

8 Brinjikji W, Murad MH, Rabinstein AA, et al. Conscious sedation versus general anesthesia during endovascular acute ischemic stroke treatment: a systematic review and meta-analysis. AJNR Am J Neuroradiol 2014;36:525-9.

9 Berkhemer OA, van den Berg LA, Fransen PSS, et al. The effect of anesthetic management during intra-arterial therapy for acute stroke in MR CLEAN. Neurology 2016;87:656-64

10 Jordan JD, Powers WJ. Cerebral autoregulation and acute ischemic stroke. Am J Hypertens 2012;25:946-50.

11 Fransen PSS, Beumer D, Berkhemer OA, et al. MR CLEAN, a multicenter randomized clinical trial of endovascular treatment for acute ischemic stroke in the Netherlands: study protocol for a randomized controlled trial. Trials 2014; 15:343

12 Froehler MT, Fifi JT, Majid A, et al. Anesthesia for endovascular treatment of acute ischemic stroke. Neurology 2012;79(Suppl 1):S167-73.

13 Lahiri S, Schlick K, Kavi T, et al. Optimizing outcomes for mechanically ventilated patients in an era of endovascular acute ischemic stroke therapy. J Intensive Care Med Published Online First: 2016 Aug 19. doi:10.1177/0885066616663168

14 Al-Mufti F, Dancour E, Amuluru K, et al. Neurocritical care of emergent large-vessel occlusion: the era of a new standard of care. J Intensive Care Med Published Online First: 2016 Jul 19. doi:10.1177/0885066616656361

15 Jellish WS, Edelstein SB. General anesthesia versus conscious sedation for the endovascular treatment of acute ischemic stroke. I Stroke Cerebrovasc Dis 2015;24:1957-60.

16 Anastasian $\mathrm{ZH}$. Anaesthetic management of the patient with acute ischaemic stroke Br J Anaesth 2014:113:ii9-16.

17 Löwhagen Hendén $P$, Rentzos A, Karlsson J-E, et al. Hypotension during endovascular treatment of ischemic stroke is a risk factor for poor neurological outcome. Stroke 2015;46:2678-80

18 Sivasankar C, Stiefel M, Miano T, et al. Anesthetic variation and potential impact of anesthetics used during endovascular management of acute ischemic stroke. J Neurointerv Surg 2016:8:1101-6.
19 Takahashi C, Brambrink A, Aziz M, et al. Association of intraprocedural blood pressure and end tidal carbon dioxide with outcome after acute stroke intervention. Neurocrit Care 2014;20:202-8.

20 Jagani $M$, Brinjikji $W$, Rabinstein $A A$, et al. Hemodynamics during anesthesia for intra-arterial therapy of acute ischemic stroke. J Neurointerv Surg 2016;8:883-8.

21 Davis MJ, Menon BK, Baghirzada LB, et al. Anesthetic management and outcome in patients during endovascular therapy for acute stroke. Anesthesiology 2012;116:396-405

22 Talke P, Sharma D, Heyer E, et al. Republished: Society for Neuroscience in Anesthesiology and Critical Care expert consensus statement: anesthetic management of endovascular treatment for acute ischemic stroke. Stroke 2014;45: e138-50.

23 Hoff RG, Van Dijk GW, Mettes S, et al. Hypotension in anaesthetized patients during aneurysm clipping: not as bad as expected? Acta Anaesth Scand 2008:52:1006-11.

24 Qureshi Al, Ezzeddine MA, Nasar A, et al. Prevalence of elevated blood pressure in 563,704 adult patients with stroke presenting to the ED in the United States. Am J Emerg Med 2007;25:32-8.

25 Wax D, Lin H-M, Leibowitz A. Invasive and concomitant noninvasive intraoperative blood pressure monitoring. Anesthesiology 2011;115:973-8.

26 Vittinghoff $E$, McCulloch C. Relaxing the rule of ten events per variable in logistic and cox regression. Am J Epidemiol 2007;165:710-18.

27 Castillo J, Leira R, García M, et al. Blood pressure decrease during the acute phase of ischemic stroke is associated with brain injury and poor stroke outcome. Stroke 2004;35:520-6

28 Leonardi-Bee J, Bath $\mathrm{P}$, Phillips $\mathrm{S}$, et al. Blood pressure and clinical outcomes in the international stroke trial. Stroke 2002;33:1315-20.

29 Whalin MK, Halenda KM, Haussen DC, et al. Even small decreases in blood pressure during conscious sedation affect clinical outcome after stroke thrombectomy: an analysis of hemodynamic thresholds. Am J Neuroradiol 2017;38:294-8

30 Schönenberger $S$, Uhlmann L, Hacke W, et al. Effect of conscious sedation vs general anesthesia on early neurological improvement among patients with ischemic stroke undergoing endovascular thrombectomy: a randomized clinical trial. JAMA 2016:316:1986-96.

31 Simonsen $C Z$, Sørensen $\mathrm{LH}$, Juul N, et al. Anesthetic strategy during endovascular therapy: general anesthesia or conscious sedation? (GOLIATH-General or Local Anesthesia in Intra Arterial Therapy) a single-center randomized trial. Int I Stroke 2016:11:1045-52. 